\title{
On the Expected Number of k-Sets*
}

\author{
Imre Bárány ${ }^{1}$ and William Steiger ${ }^{2}$ \\ ${ }^{1}$ Mathematical Institute, The Hungarian Academy of Sciences, \\ Pf. 127, H-1364 Budapest, Hungary \\ h2923bar@ella.hu \\ ${ }^{2}$ Department of Computer Science, Rutgers University, \\ Piscataway; NJ 08903, USA \\ steiger@cs.rutgers.edu
}

\begin{abstract}
Given a set $S$ of $n$ points in $R^{d}$, a subset $X$ of size $d$ is called a $k$-simplex if the hyperplane aff $(X)$ has exactly $k$ points on one side. We study $E_{d}(k, n)$, the expected number of $\mathrm{k}$-simplices when $S$ is a random sample of $n$ points from a probability distribution $P$ on $R^{d}$. When $P$ is spherically symmetric we prove that $E_{d}(k, n) \leq c n^{d-1}$. When $P$ is uniform on a convex body $K \subset R^{2}$ we prove that $E_{2}(k, n)$ is asymptotically linear in the range $c n \leq k \leq n / 2$ and when $k$ is constant it is asymptotically the expected number of vertices on the convex hull of $S$. Finally, we construct a distribution $P$ on $R^{2}$ for which $E_{2}((n-2) / 2, n)$ is $c n \log n$.
\end{abstract}

\section{Introduction and Summary}

Let $X$ be a set of $n$ points in $R^{d}$ in general position. The $\operatorname{simplex} \operatorname{conv}(S)$ (when $S \subset X$ and $|S|=d)$ is called a $k$-simplex if $X$ has exactly $k$ points on one side of the hyperplane aff(S). A k-simplex is an (n-d-k)-simplex as well. Although this should not cause any confusion we always try to have $k \leq n-d-k$. In two dimensions a $\mathrm{k}$-simplex is called a $k$-segment.

Write $e_{d}(k, n)$ for the maximal number of $\mathrm{k}$-simplices over all configurations $X$ of $n$ points in $R^{d}$. Most of the previous work has focused on $e_{d}(k, n)$ because of its connection with $k$-sets. A subset $Y \subset X$ of size $k$ is called a k-set if $Y$ and $X \backslash Y$ are separated by a hyperplane. The question is: how many k-sets may a set $X$

\footnotetext{
* The authors express gratitude to the NSF DIMACS Center at Rutgers and Princeton. The research of I. Bárány was supported in part by Hungarian National Science Foundation Grants 1907 and 1909, and W. Steiger's research was supported in part by NSF Grants CCR-8902522 and CCR-9111491.
} 
possess? It is easy to translate an upper bound for $e_{d}(k, n)$ into an upper bound on k-sets.

Clearly, $O\left(n^{d}\right)$ provides a trivial upper bound for $e_{d}(k, n)$. When $d=2$, nontrivial bounds were obtained by Lovász [15] for halving sets ( $n$ even, $k=n / 2$ ), and later, for general $k \leq n / 2$, by Erdós et al. [12]. A simple construction gives a set $S$ with $n \log k$ k-sets, while a counting argument shows that $e_{2}(k, n)=O(n \sqrt{k})$. These bounds were rediscovered several times, for example by Edelsbrunner and Welzl [11], but had not been improved until Pach et al. [17] reduced the bound to $n \sqrt{k} / \log * k$. Papers [1], [13], and [22] contain results related to the study of $e_{2}(k, n)$.

Raimund Seidel (see [10]) extended the Lovász lower bound construction to $d=3$ and showed that $e_{3}(k, n)=\Omega(n k \log (k+1))$. The argument may be applied inductively giving $e_{d}(k, n)=\Omega\left(n k^{d-2} \log (k)\right)$.

A nontrivial upper bound for $d=3$ was recently obtained by Bárány et al. [5]. They showed that $e_{3}(n / 2, n)=n^{3-\varepsilon}$, where $\varepsilon>0$ is some small constant. This, in turn, was improved by Aronov et al. [2] to $O\left(n^{8 / 3} \log ^{5 / 3} n\right)$. Dey and Edelsbrunner [9] have been able to remove the logarithmic factors from this bound. Recently, a nontrivial upper bound for $d>3$ was established via a result of Živaljević and Vrećica [23]. They proved a colored version of Tverberg's theorem which now implies that $O\left(n^{d-\varepsilon_{d}}\right)$ is an upper bound for halving sets in $R^{d}, \varepsilon_{d}>0$ being a small constant depending on $d$.

It appears likely that the truth is near the lower bound. Support comes from the fact that in "typical" cases there are relatively few $\mathrm{k}$-sets. In this paper we study $E_{d}(k, n)$, the expected number of k-simplices when $X$ is a sample of $n$ random points from a probability measure $P$ on $R^{d}$. When there is no confusion we write $E(k, n)$. The following derivation gives an expression for $E_{d}(k, n)$ that we use throughout. Pick $d$ points $x_{1}, \ldots, x_{d}$ independently, according to $P$. Write $l$ for the hyperplane $\operatorname{aff}\left(x_{1}, \ldots, x_{d}\right)$. We assume throughout that $P$ vanishes on every hyperplane so $l$ is well defined with probability one. (In particular, $P$ is nonatomic.) Write $l^{+}$and $l^{-}$for the open half-spaces on the right and left of $l$, respectively, and set $F(l)=\min \left(P\left(l^{+}\right), P\left(l^{-}\right)\right)$, the probability content cut off by $l$. The random variable $F(l)$ has a distribution function

$$
G(t)=P(F(l) \leq t)
$$

which determines $E_{d}(k, n)$ in the following way. Given a sample $X=\left\{x_{1}, \ldots, x_{n}\right\}$ from $P$, the expected number of $\mathrm{k}$-simplices is

$$
\begin{aligned}
E_{d}(k, n) & =\sum_{1 \leq i_{1}<\cdots<i_{d} \leq n} \operatorname{Prob}\left[\text { there are } k \text { points on one side of aff }\left(x_{i_{1}}, \ldots, x_{i_{d}}\right)\right] \\
& =\left(\begin{array}{c}
n \\
d
\end{array}\right)\left(\begin{array}{c}
n-d \\
k
\end{array}\right) \int_{0}^{1 / 2}\left[t^{k}(1-t)^{n-d-k}+(1-t)^{k} t^{n-d-k}\right] d G(t) .
\end{aligned}
$$

Our first result is a simple one about spherically symmetric distributions (the definition is given in Section 2). 
Theorem 1. For a spherically symmetric distribution we have $E_{d}(k, n) \leq c_{1} n^{d-1}, c_{1}$ being a constant depending only on d.

Next we deal with the case where $P$ is the uniform distribution on a compact, convex body $K \subset R^{2}$. We assume that $\operatorname{Area}(K)=1$ so that $P$ coincides with the restriction of Lebesgue measure to $K$. Define $v: K \mapsto R$ by

$$
v(x)=\inf \{\operatorname{Area}(K \cap H): x \in H, H \text { is a half-plane }\}
$$

and $A(t)=A_{K}(t)=\operatorname{Area}\{x \in K: v(x) \leq t\}$. The properties of $v$ and $A(t)$ have been studied in [3], [6], and [21]. Here we prove that $G$ is differentiable and that $G^{\prime}(t)$ is essentially equal to $A(t)$. This helps establish the following theorem.

Theorem 2. There are absolute constants $c_{2}$ and $c_{3}$ such that, for the uniform distribution over any convex set in the plane,

$$
c_{2} n A\left(\frac{k+1}{n}\right) \leq E_{2}(k, n) \leq c_{3} n A\left(\frac{k+1}{n}\right)
$$

for every sufficiently large $n$ and every $k=0,1, \ldots,\lfloor(n-2) / 2\rfloor$.

Sometimes we express the relation in (4) as

$$
E_{2}(k, n) \sim n A\left(\frac{k+1}{n}\right)
$$

Remark 1. Since $t \leq A(t)$, we have $c_{4} \leq A(t) \leq 1$ when $t \geq c_{4}>0$. Theorem 2 then shows that when $\frac{1}{2} \geq k / n \geq c_{4}$,

$$
E_{2}(k, n) \sim n
$$

The behavior of $A(t)$ (for small $t$ ) is given by Theorem 7 of [6]

$$
c_{5} t \log \frac{1}{t} \leq A(t) \leq c_{6} t^{2 / 3}
$$

Schütt and Werner [21] show that for a function $f(t)$ with $c_{5} t \log (1 / t) \leq$ $f(t) \leq c_{6} t^{2 / 3}$ (and some additional properties) there is a convex set $K=K_{f} \subset R^{2}$ of area 1 such that $A_{K}(t) \sim f(t)$. This shows that not only does

$$
c_{5}(k+1) \log \frac{n}{k+1} \leq E_{2}(k, n) \leq c_{6} n\left(\frac{k+1}{n}\right)^{2 / 3}
$$


hold for $P$ uniform on a convex body, but also, for (almost) any function between these bounds, there is a convex body $K$ with $E_{2}(k, n)$ behaving like that function.

The special case $k=0$ is interesting. Then $E_{2}(k, n)$ equals the expected number of edges of $\operatorname{conv}(X)$, which was known to behave like $A(1 / n)$ (see [6]). So Theorem 2 says that $E_{2}(k, n)$ behaves like the expected number of edges of $\operatorname{conv}(X)$ when $k$ is a constant, and like $n$ when $k / n \geq t_{0}$.

Finally, we give an example of a distribution for which $E_{2}(k, n)$ is large. We consider the case $k=(n-2) / 2$ ( $n$ even), that is, the expected number of halving segments. We give a distribution $P_{n}$ such that

$$
E_{2}\left(\frac{m-2}{2}, m\right) \geq c_{7} m \log m
$$

whenever the sample size $m$ is within a constant factor of $n$. Then, using $P_{n}$, we describe a distribution $P$ for which

$$
E_{2}\left(\frac{n-2}{2}, n\right) \geq c_{8} n \log n
$$

Finally, we point out the abstract of [7], where one of the present results was announced, but with an erroneous proof. This is one of the reasons we take some care in establishing the simple statements about $E_{d}$. The methods are familiar in geometric probability and integral geometry (see [4], [16], and [19]). Nevertheless, the results seem to be the first ones concerning $E_{d}$ and in view of the fact that $\mathrm{k}$-sets have applications in computational geometry and machine learning [14], [18], we feel that these theorems are useful and interesting.

\section{Spherically Symmetric Continuous Distributions}

Suppose that $P$ has a density function $g: R^{d} \rightarrow R$ that only depends on $|x|$, the distance from $x \in R^{d}$ to the origin. We say that such a $P$ is spherically symmetric. This defines another function $f: R_{+} \rightarrow R$ by $f(r)=g(|x|)$ when $r=|x|$.

Proof of Theorem 1. Set $\kappa_{d-1}=v l_{d-1}\left(S^{d-1}\right)$. Clearly,

$$
1=\int_{R^{d}} g(x) d x=\int_{u \in S^{d-1}} \int_{r=0}^{\infty} f(r) r^{d-1} d r d u=\kappa_{d-1} \int_{0}^{\infty} f(r) r^{d-1} d r
$$


Now let $H(t)$ be an open half-space with probability content $t, 0<t \leq \frac{1}{2}$, and write $p=p(t)$ for the distance (from the origin) to $\Pi$, the bounding hyperplane of $H(t)$. Then

$$
t=\int_{H(t)} g(x) d x=\int_{r=p}^{\infty} \int_{y \in R^{d-1}} f\left(\sqrt{r^{2}+|y|^{2}}\right) d y d r
$$

where $r$ is the length of the component of $x$ parallel to $u$ and $y=x-u r, u$ denoting the unit normal to $\Pi$.

Claim 1. $G(t+\Delta t)-G(t) \leq c_{9} \Delta t$

Theorem 1 follows immediately because, from (2),

$$
\begin{aligned}
E_{d}(k, n) & \leq\left(\begin{array}{l}
n \\
d
\end{array}\right)\left(\begin{array}{c}
n-d \\
k
\end{array}\right) \int_{0}^{1 / 2}\left[t^{k}(1-t)^{n-d-k}+(1-t)^{k} t^{n-d-k}\right] c_{9} d t \\
& \leq c_{10} n^{d-1}
\end{aligned}
$$

the last inequality is a consequence of the well-known fact that

$$
(m+1)\left(\begin{array}{c}
m \\
j
\end{array}\right) \int_{0}^{1} t^{j}(1-t)^{m-j} d t=1
$$

Proof of Claim 1. We use the Blaschke-Petkantschin formula (see p. 201 of [20]) which says that

$$
d x_{1} \cdots d x_{d}=d ! v o l_{d-1}\left(\operatorname{conv}\left\{y_{1}, \ldots, y_{d}\right\}\right) d y_{1} \cdots d y_{d} d u d r
$$

where the points $x_{1}, \ldots, x_{d}$ lie in the hyperplane $u x=r$ with $u \in S^{d-1}$, the unit sphere in $R^{d}$ and $r>0$, and $y_{i}=x_{i}-r u$. With this fact,

$$
\begin{aligned}
G(t+\Delta t)-G(t)= & \int \cdots \int_{t<F(t) \leq t+\Delta t} g\left(x_{1}\right) \cdots g\left(x_{d}\right) d x_{1} \cdots d x_{d} \\
= & \int_{r=p(t+\Delta t)}^{p(t)} \int_{u \in S^{d-1}} \int_{y_{1}} \cdots \int_{y_{d}} f\left(\sqrt{r^{2}+\left|y_{1}\right|^{2}} \cdots f\left(\sqrt{r^{2}+\left|y_{d}\right|^{2}}\right)\right. \\
& \times d ! v o l_{d-1}\left(\operatorname{conv}\left\{y_{1}, \ldots, y_{d}\right\}\right) d y_{1} \cdots d y_{d} d u d r \\
= & d ! \kappa_{d-1} \int_{p(t+\Delta t)}^{p(t)} \int_{R^{d-1}} \cdots \int_{R^{d-1}} f\left(\sqrt{r^{2}+\left|y_{1}\right|^{2}}\right) \cdots f\left(\sqrt{r^{2}+\left|y_{d}\right|^{2}}\right) \\
& \times v o l_{d-1}\left(\operatorname{conv}\left\{y_{1}, \ldots, y_{d}\right\}\right) d y_{1} \cdots d y_{d} d r .
\end{aligned}
$$


Notice that the innermost $d$ integrals here denote the expectation of the volume of $\operatorname{conv}\left\{y_{1}, \ldots, y_{d}\right\}$ when the points $y_{1}, \ldots, y_{d}$ are distributed on the hyperplane $H=\{x: u x=r\}$ according to density $f\left(\sqrt{r^{2}+|y|^{2}}\right)$. This is, again, a spherically symmetric distribution in the hyperplane $H$ with center $r u$ which we take for the origin of $H$ and denote by $\overline{0}$. The signed volume of $\operatorname{conv}\left\{y_{1}, \ldots, y_{d}\right\}$ is

$$
\frac{1}{(d-1) !} \operatorname{det}\left(\begin{array}{ccc}
1 & \cdots & 1 \\
y_{1} & \cdots & y_{d}
\end{array}\right)
$$

but

$$
\operatorname{det}\left(\begin{array}{ccc}
1 & \cdots & 1 \\
y_{1} & \cdots & y_{d}
\end{array}\right)=\operatorname{det}\left(\begin{array}{cccc}
1 & 1 & \cdots & 1 \\
0 & y_{2} & \cdots & y_{d}
\end{array}\right)+\cdots+\operatorname{det}\left(\begin{array}{cccc}
1 & \cdots & 1 & 1 \\
y_{1} & \cdots & y_{d-1} & 0
\end{array}\right)
$$

Consequently, with unsigned volumes,

$$
\operatorname{vol}\left(\operatorname{conv}\left\{y_{1}, \ldots, y_{d}\right\}\right) \leq \sum_{i=1}^{d} \operatorname{vol}\left(\operatorname{conv}\left\{\left\{y_{1}, \ldots, y_{d}, \overline{0}\right\} \backslash\left\{y_{i}\right\}\right\}\right)
$$

Since every term on the right-hand side has the same expectation,

$$
E\left[\operatorname{vol}\left(\operatorname{conv}\left\{y_{1}, \ldots, y_{d}\right\}\right)\right] \leq d E\left[\operatorname{vol}\left(\operatorname{conv}\left\{\overline{0}, y_{1}, \ldots, y_{d-1}\right\}\right)\right]
$$

Moreover,

$$
\operatorname{vol}\left(\operatorname{conv}\left\{\overline{0}, y_{1}, \ldots, y_{d-1}\right\}\right)=\frac{1}{(d-1) !}\left|\operatorname{det}\left(y_{1}, \ldots, y_{d-1}\right)\right| \leq \frac{1}{(d-1) !} \prod_{i=1}^{d-1}\left|y_{i}\right|
$$

by Hadamard's inequality. This way we get

$$
\begin{aligned}
G(t+\Delta t)-G(t) \leq & d^{2} \kappa_{d-1} \int_{r=p(t+\Delta t)}^{p(t)}\left[\prod_{i=1}^{d-1} \int_{y_{i}} f\left(\sqrt{r^{2}+\left|y_{i}\right|^{2}}\right)\left|y_{i}\right| d y_{i}\right] \\
& \times \int_{y_{d}} f\left(\sqrt{r^{2}+\left|y_{d}\right|^{2}}\right) d y_{d} d r .
\end{aligned}
$$


From (6),

$$
\begin{aligned}
\int_{R^{d-1}} f\left(\sqrt{r^{2}+y_{i}^{2}}\right)\left|y_{i}\right| d y_{i} & =\int_{u \in S^{d-2}} \int_{s=0}^{\infty} f\left(\sqrt{r^{2}+s^{2}}\right) s \cdot s^{d-2} d s d u \\
& =\kappa_{d-2} \int_{s=0}^{\infty} f\left(\sqrt{r^{2}+s^{2}}\right) s^{d-1} d s \\
& =\kappa_{d-2} \int_{q=r}^{\infty} f(q)\left(q^{2}-r^{2}\right)^{(d-1) / 2} \frac{q d q}{\sqrt{q^{2}-r^{2}}} \\
& \leq \kappa_{d-2} \int_{q=r}^{\infty} f(q) q^{d-1} d q \leq \frac{\kappa_{d-2}}{\kappa_{d-1}} .
\end{aligned}
$$

By (7)

$$
\int_{r=p(t+\Delta t)}^{p(t)} \int_{R^{d-1}} f\left(\sqrt{r^{2}+\left|y_{d}\right|^{2}}\right) d y_{d} d r=\Delta t
$$

and therefore

$$
G(t+\Delta t)-G(t) \leq d^{2} \kappa_{d-2}\left(\frac{\kappa_{d-2}}{\kappa_{d-1}}\right)^{d-1} \Delta t
$$

\section{Uniform Distribution on a Convex Set}

Let $K \subset R^{2}$ be a convex set with $\operatorname{Area}(K)=1$. We are interested in $E_{2}(k, n)$ when $P$ is the Lebesgue measure restricted to $K$. Since $E_{2}$ is invariant under (nondegenerate) affine transformations of $K$ we may assume that $K$ is in "normal position," i.e., that

$$
r B^{2} \subset K \subset 2 r B^{2}
$$

where $B^{2}$ is the unit disk, centered at the origin, and $r$ is a universal constant (in fact $r=3^{-3 / 4}$, but we do not need this precision). The existence of the "normal position" follows from that of the Löwner-John ellipsoid [8].

It is more convenient to work with the directed version of (2). So let $l=\overrightarrow{x y}$ denote the line directed from $x$ to $y$. Write $F(l)$ for the probability content of the half-plane $l^{+}$on the right of $l$; this is equal to the area of $K \cap l^{+}$. Set $G(t)=$ $\operatorname{Prob}[F(l) \leq t]$. Then (2) becomes

$$
E_{2}(k, n)=\left(\begin{array}{l}
n \\
2
\end{array}\right)\left(\begin{array}{c}
n-2 \\
k
\end{array}\right) \int_{0}^{1} t^{k}(1-t)^{n-2-k} d G(t) .
$$

We need some further notation. Given $\varphi \in[0,2 \pi]$ and $t \in(0,1)$ there is a unique directed line $l(\varphi, t)$ with direction $\varphi$ that has $F(l)=t . l(\varphi, t)$ is clearly continuous in 
both variables and can be extended to $t=0$ and $t=1$. In that case $l(\varphi, 0)$, say, denotes the directed line that has $K$ on its left and supports $K$. The line $l(\varphi, t)$ has signed distance $p(\varphi, t)$ from the origin so that $p(\varphi, t) \geq 0$ if the origin is on the left of $l(\varphi, t)$ (or on $l(\varphi, t)$ itself) and $p(\varphi, t)<0$ otherwise. Also, for any $\varphi \in[0,2 \pi]$, $p(\varphi, t) \in[p(\varphi, 1) . p(\varphi, 0)]$.

The directed line having direction $\varphi$ and at signed distance $p$ from the origin cuts $K$ into two parts of area $t(\varphi, p)$ on its right and $1-t(\varphi, p)$ on its left. Define $\psi(\varphi, t)$ as the length of the chord $l(\varphi, t) \cap K$. Clearly, $p=p(\varphi, t(\varphi, p))$ identically. It is evident that $p \mapsto \psi(\varphi, t(\varphi, p))$ is a concave function on $[p(\varphi, 1), p(\varphi, 0)]$.

Recall the definition of $v(x)$ from (3). We write $K(t)=\{x \in K: v(x) \leq t\}$ and $A(t)$ for its area.

Theorem 3. $G(t)$ is differentiable when $t \in(0,1)$ and

$$
G^{\prime}(t)=\frac{1}{6} \int_{0}^{2 \pi} \psi^{2}(\varphi, t) d \varphi
$$

Theorem 4. As $t \rightarrow 0$,

$$
G^{\prime}(t)=\frac{4}{3} A(t)(1+o(1))
$$

We mention here that $G(t) \sim t A(t)$ is proved in [3]. Theorems 3 and 4 establish a different and apparently more subtle property of the function $G$. We need:

Lemma 1. For each $t \in(0,1)$ there is a constant $C_{t}$ such that

$$
|\psi(\varphi, t)-\psi(\varphi, u)| \leq C_{t}|t-u|
$$

for all $\varphi \in[0,2 \pi]$ and $u \in[0,1]$. In fact, $C_{t}=8 r / \min (t, 1-t)$.

The proof is straightforward using the normal position and the following easy facts (refer to Fig. 1):

1. The chord function $p \mapsto \psi(\varphi, t(\varphi, p))$ is concave in $p$.

2. For all $s \in(0,1), 4 r(p(\varphi, 0)-p(\varphi, s)) \geq s$ and $4 r(p(\varphi, s)-p(\varphi, 1)) \geq 1-s$.

3. $\psi(\varphi, s)(p(\varphi, t)-p(\varphi, u)) \leq 2(u-t)$ if $s=u$ or $s=t$.

We omit the details. 


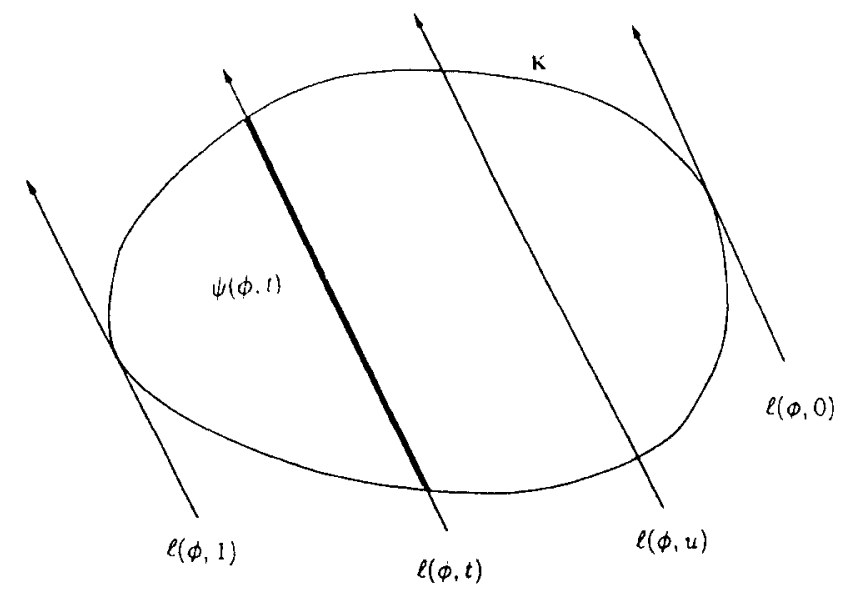

Fig. 1. The chord function $\psi(\varphi, t)=|l(\varphi, t) \cap K|$.

Proof of Theorem 3. Write $l=\overrightarrow{x y}$. By the Blaschke-Petkantschin formula,

$$
\begin{aligned}
G(t+\Delta t)-G(t) & =\operatorname{Prob}[F(l) \in[t, t+\Delta t)] \\
& =\int_{t \leq F(\vec{x})<t+\Delta t} \int 1 d x d y \\
& =\int_{0}^{2 \pi} \int_{p(\varphi, t+\Delta t)}^{p(\varphi, t)} \iint_{\tilde{x}<\bar{y} \in K \cap l}|\bar{x}-\bar{y}| d \bar{x} d \bar{y} d p d \varphi .
\end{aligned}
$$

An elementary computation reveals that

$$
\iint_{\bar{x}<\bar{y} \in \mathbf{K} \cap l}|\bar{x}-\bar{y}| d \bar{x} d \bar{y}=\frac{1}{6} \chi^{3}(l),
$$

where $\chi(l)=\psi(\varphi, t(\varphi, p))$ is the length of the chord $K \cap l$. So

$$
\begin{aligned}
G(t+\Delta t)-G(t)= & \frac{1}{6} \int_{0}^{2 \pi} \int_{p(\varphi, t+\Delta t)}^{p(\varphi, t)} \psi^{3}(\varphi, t(\varphi, p)) d p d \varphi \\
= & \frac{1}{6} \int_{0}^{2 \pi} \psi^{2}(\varphi, t) \int_{p(\varphi, t+\Delta t)}^{p(\varphi, t)} \psi(\varphi, t(\varphi, p)) d p d \varphi \\
& +\frac{1}{6} \int_{0}^{2 \pi} \int_{p(\varphi, t+\Delta t)}^{p(\varphi, t)}\left[\psi^{2}(\varphi, t(\varphi, p))-\psi^{2}(\varphi, t)\right] \psi(\varphi, t(\varphi, p)) d p d \varphi
\end{aligned}
$$


The first term here equals $\frac{1}{6} \int_{0}^{2 \pi} \psi^{2}(\varphi, t) d \varphi \Delta t$ since trivially (again, see Fig. 1)

$$
\Delta t=\int_{p(\psi, t+\Delta t)}^{p(\psi, t)} \psi(\varphi, t(\varphi, p)) d p .
$$

Therefore

$$
\begin{aligned}
& \left|\frac{G(t+\Delta t)-G(t)}{\Delta t}-\frac{1}{6} \int_{0}^{2 \pi} \psi^{2}(\varphi, t) d \varphi\right| \\
& \quad \leq \frac{1}{6 \Delta t} \int_{0}^{2 \pi} \int_{p(\varphi, t+\Delta t)}^{p(\varphi, t)}\left|\psi^{2}(\varphi, t(\varphi, p))-\psi^{2}(\varphi, t)\right| \psi(\varphi, t(\varphi, p)) d p d \varphi \\
& \quad \leq \frac{1}{6 \Delta t} \int_{0}^{2 \pi} \int_{p(\varphi, t+\Delta t)}^{p(\varphi, t)} 8 r C_{t} \Delta t \psi(\varphi, t(\varphi, p)) d p d \varphi \\
& \quad=\frac{8 r \pi}{3} C_{i} \Delta t
\end{aligned}
$$

where we used Lemma 1 in the last inequality and (11) in the last equality.

Remark 2. We point out that, for $\frac{1}{2} \geq t \geq t_{0}>0$,

$$
c_{11} \leq G^{\prime}(t) \leq c_{12}
$$

The upper bound is trivial from Theorem 3 because $\psi$ is bounded. For the lower bound it is enough to see that $\psi(\varphi, t) \geq c_{13} t$. This follows easily from the normal position of $K$.

Before the proof of Theorem 4 we need some preparation. The body

$$
K(v \geq t)=\{x \in K: v(x) \geq t\}
$$

is clearly convex. We assume $t \leq t_{0} \leq 0.01$, say, and then $K(v \geq t)$ is nonempty as well. Thus the boundary of $K(v \geq t)$ is a convex curve $V(t)$ with left and right tangents at every $z \in V(t)$. These tangents coincide at all but countably many $z \in V(t)$.

Fix $t \in\left(0, t_{0}\right]$. Given $\varphi \in[0,2 \pi)$ let $\lambda(\varphi, t)$ be the unique directed line (with direction $\varphi$ ) that is a supporting line to $K(v \geq t)$ and has $K(v \geq t)$ on its left. $\lambda(\varphi, t)$ has exactly one point (to be denoted by $z(\varphi, t)$ ) in common with $K(v \geq t)$ since, as is proved in [3], $V(t)$ contains no line segment. Call the angle $\varphi$ regular if $\lambda(\varphi, t)$ is tangent (left, right, or both) to the curve $V(t)$ at $z(\varphi, t)$. Write $R$ for the set of regular angles in $[0,2 \pi]$ and $N R$ for its complement. It is not difficult to see that $R$ is a closed set. Therefore $N R$ is a countable union of open intervals; the point in the proof of Theorem 4 is that the total length of these intervals is $O(t)$. 
Recall that $l(\varphi, t)$ is a directed line that cuts off area $t$ from $K$. It follows from the proof of Lemma $\mathrm{G}$ in [3] that if $\varphi$ is regular, then $\lambda(\varphi, t)$ and $l(\varphi, t)$ coincide and $z(\varphi, t)$ is the midpoint of the chord $K \cap l(\varphi, t)$. Finally, let $L(\varphi, t)$ be the length of the segment connecting $z(\varphi, t)$ to the last point on $\lambda(\varphi, t)$ in $K$. Observe that, for a regular angle, $L(\varphi, t)=\frac{1}{2} \psi(\varphi, t)$.

We omit the simple proof of the following.

Claim 2. Area $(K(v \geq t))=\frac{1}{2} \int_{0}^{2 \pi} L^{2}(\varphi, t) d \varphi$.

Lemma 2. The total length of the intervals in $N R$ is $O(t)$.

Proof. Assume that $\varphi$ is nonregular and let $\varphi^{+}$and $\varphi^{-}$be the direction of the left and right tangents $l^{+}$and $l^{-}$to $V(t)$ at $z(\varphi, t)$. Since $\varphi^{+}\left(\varphi^{-}\right)$are regular, $z(\varphi, t)$ is the midpoint of the corresponding chords which we denote by $u^{+} v^{+}$and $u^{-} v^{-}$, as is shown in Fig. 2. Then $u^{-} u^{+}$and $v^{-} v^{+}$span parallel lines. Let $S$ be the strip between them. Clearly,

$$
\operatorname{Area}\left(r B^{2} \backslash S\right) \leq \operatorname{Area}(K \backslash S) \leq 2 t
$$

An elementary computation reveals that the width of $S$ is at least $2 r-$ $\left(t^{2} /(2 r)\right)^{1 / 3}>0.8$, so

$$
\min \left(\left|u^{-}-v^{-}\right|,\left|u^{+}-v^{+}\right|\right) \geq 0.8
$$

Moreover,

$$
1=\operatorname{Area}(K) \leq 2 t+\frac{1}{2} \operatorname{diam}^{2}(K)\left(\pi-\left(\varphi^{+}-\varphi^{-}\right)\right)
$$

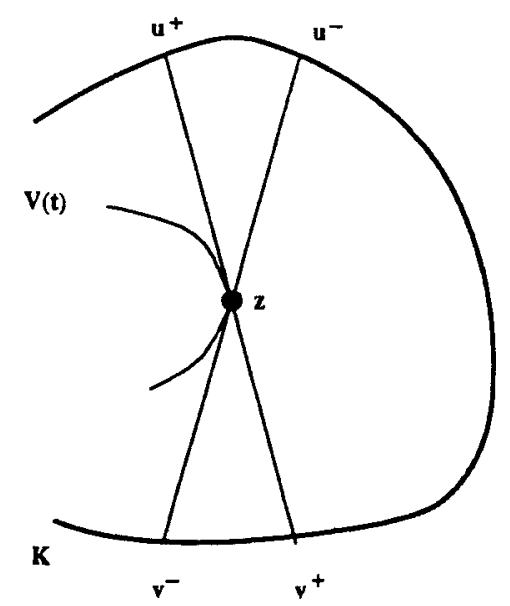

Fig. 2. Tangents at a nonregular point $z=z(\varphi, t)$ on $V(t)$. 
because both lines $l^{+}$and $l^{-}$cut off a cap from $K$ of area $t$, and the remainder is contained in a circular sector with center $z(\varphi, t)$, radius equal to $\operatorname{diam}(K) \leq 4 r$, and angle $\pi-\left(\varphi^{+}-\varphi^{-}\right)$. Consequently (see Fig. 2),

$$
\varphi^{+}-\varphi^{-} \leq \pi-\frac{1-2 t}{8 r^{2}}
$$

so $\varphi^{+}-\varphi^{-}$is separated from $\pi$. On the other hand,

$$
\begin{aligned}
t \geq \operatorname{Area}\left(\operatorname{conv}\left\{u^{-}, u^{+}, v^{-}\right\}\right) & =\frac{1}{2}\left|u^{+}-v^{+}\right| \frac{1}{2}\left|u^{-}-v^{-}\right| \sin \left(\varphi^{+}-\varphi^{-}\right) \\
& \geq \frac{1}{4}(0.8)^{2} \sin \left(\varphi^{+}-\varphi^{-}\right)>0.16 \sin \left(\varphi^{+}-\varphi^{-}\right)
\end{aligned}
$$

proving that

$$
\varphi^{+}-\varphi^{-} \leq 8 t
$$

This shows that NR contains only "short" intervals.

Because $t<0.01$ a smaller disk, $0.8 r B^{2}$, is contained in $K(v \geq t)$. Call the points $z(\varphi, t)$ nonregular if $\varphi \in N R$, and the other points of $V(t)$ regular. Observe that there are only countably many nonregular points, each one corresponding to an interval from $N R$. Choose a regular point $z\left(\varphi_{1}, t\right)$ and take $\varphi_{1}$ to be 0 . We are going to construct, by induction, a sequence of regular points $z_{1}=z\left(\varphi_{1}, t\right), \ldots, z_{m}=z\left(\varphi_{m}, t\right)$ with $\varphi_{1}<\varphi_{2}<\cdots<\varphi_{m}<2 \pi$. Assume $\varphi_{1}, \ldots, \varphi_{i}$ have already been constructed. Pick a regular point $z=z(\varphi, t)$ with $\varphi>\varphi_{i}$ so that $\left|z-z_{i}\right| \in[0.19,0.20]$. Such a point clearly exists. Further, it can be chosen so that $\varphi-\varphi_{i} \leq \frac{3}{4} \pi$, as can easily be seen from $0.8 r B^{2} \subset K(v \geq t)$. Now if $\varphi-\varphi_{i} \leq \pi / 2$, then we define $\varphi_{i+1}=\varphi$ and $z_{i+1}=z$. However, if not, then define $\varphi_{i+1}$ to be a regular angle very close to $\left(\varphi+\varphi_{i}\right) / 2$ and set $\varphi_{i+2}=\varphi$. Since the intervals in $N R$ are shorter than $8 t \leq 0.08$ we have $\varphi_{i+1}-\varphi_{i} \leq \pi / 2$ and $\varphi_{i+2}-\varphi_{i+1} \leq \pi / 2$. We stop when the next $\varphi, \varphi_{m+1}$ is larger than $2 \pi$.

It is easy to see now that $m \leq 35$. Indeed, $\varphi-\varphi_{i}>\pi / 2$ can happen at most three times, and in the other cases $\left|z_{i+1}-z_{i}\right| \geq 0.19$. As the perimeter of $V(t)$ is at most $4 \pi r$ we get $m \leq 3+(4 \pi r) / 0.19 \leq 35$.

Consider now the counterclockwise arc $A_{i}$ connecting $z_{i}$ to $z_{i+1}$ on $V(t)$. Let $w_{1}$ and $w_{2}$ be two nonregular points on $A_{i}, w_{1}$ having the left tangent direction $\psi_{1}$ and $w_{2}$ having the right tangent direction $\psi_{2}$, with $0<\psi_{2}-\psi_{1}$. The inequality $\psi_{2}-\psi_{1}<\pi / 2$ is automatically satisfied since, by the construction, $\varphi_{i+1}-\varphi_{i} \leq \pi / 2$.

Claim 3. $\psi_{2}-\psi_{1}<16 t$.

Proof (see Fig. 3). If $w_{1}=w_{2}$, then this follows from (14). Otherwise, let $w$ be the intersection of the tangents at $w_{1}$ and $w_{2}$. Observe that the angle $w_{1} w w_{2}$ is at least $\pi / 2$ (since $\psi_{2}-\psi_{1} \leq \pi / 2$ ), so

$$
\left|w-w_{2}\right| \leq\left|w_{1}-w_{2}\right| \leq\left|z_{i}-z_{i+1}\right| \leq 0.20 \text {. }
$$




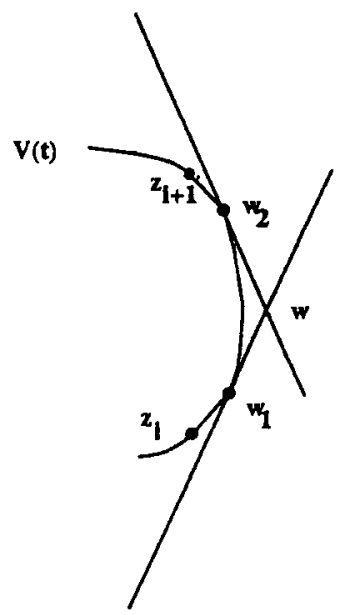

Fig. 3. Left and right tangents at nonregular points $w_{1}$ and $w_{2}$.

Again, we used $\varphi_{i+1}-\varphi_{i} \leq \pi / 2$. Moreover, writing $u_{2} v_{2}$ for the tangent chord in direction $\psi_{2}$ and $u_{1} v_{1}$ for the other tangent chord,

$$
\begin{aligned}
t \geq \operatorname{Area}\left(\operatorname{conv}\left\{u_{1}, u_{2}, v_{1}\right\}\right) & =\frac{1}{2}\left|w-u_{2}\right|\left|u_{1}-v_{1}\right| \sin \left(\psi_{2}-\psi_{1}\right) \\
& \geq \frac{1}{2}\left(\frac{1}{2}\left|v_{2}-u_{2}\right|-0.20\right)\left|u_{1}-v_{1}\right| \sin \left(\psi_{2}-\psi_{1}\right) \\
& \geq \frac{1}{2}(0.20)(0.8) \sin \left(\psi_{2}-\psi_{1}\right)=0.08 \sin \left(\psi_{2}-\psi_{1}\right),
\end{aligned}
$$

where we used (13) as well.

Proof of Theorem 4. For a regular direction, $L(\varphi, t)=\frac{1}{2} \psi(\varphi, t)$. Then

$$
\begin{aligned}
G^{\prime}(t) & =\frac{1}{6} \int_{0}^{2 \pi} \psi^{2}(\varphi, t) d \varphi \\
& =\frac{1}{6}\left[\int_{R} \psi^{2}(\varphi, t) d \varphi+\int_{N R} \psi^{2}(\varphi, t) d \varphi\right] \\
& =\frac{1}{6}\left[\int_{0}^{2 \pi} 4 L^{2}(\varphi, t) d \varphi+\int_{N R}\left(\psi^{2}(\varphi, t)-4 L^{2}(\varphi, t)\right) d \varphi\right] \\
& =\frac{4}{3} A(t)+\frac{1}{6} \int_{N R}\left(\psi^{2}(\varphi, t)-4 L^{2}(\varphi, t)\right) d \varphi
\end{aligned}
$$

If all directions are regular, we are finished. Otherwise

$$
\begin{aligned}
\left|G^{\prime}(t)-\frac{4}{3} A(t)\right| & \leq \frac{1}{6} \int_{N R}\left|\psi^{2}(\varphi, t)-4 L^{2}(\varphi, t)\right| d \varphi \\
& \leq \frac{16 r^{2}}{6} \operatorname{meas}(N R) \leq c_{14} t
\end{aligned}
$$


According to [6], $A(t) \geq c t \log (1 / t)$ for some absolute constant $c$, so we get

$$
G^{\prime}(t)=\frac{4}{3} A(t)\left(1+O\left(\frac{1}{\log (1 / t)}\right)\right)
$$

Theorem 2 follows easily from Theorems 3 and 4 using some properties of $A(t)$, namely:

1. $1 \geq A(t) \geq 0$ and $A(t)$ is monotone increasing.

2. $A(\alpha t) \leq c_{15} \alpha^{2} A(t)$, if $\alpha \geq 1$ and $t>0$ (see [6]).

Proof of Theorem 2. When $k=0, E_{2}(k, n)$ is the expected number of edges (or vertices) of the convex hull and this case is covered in [6]. So assume $k \geq 1$. It follows from properties 1 and 2 above that $A((k+1) / n) \sim A(k /(n-2))$. We write $m=n-2$ to simplify the notation. By Theorem 3

$$
E_{2}(k, m+2)=\left(\begin{array}{c}
m+2 \\
2
\end{array}\right)\left(\begin{array}{l}
m \\
k
\end{array}\right) \int_{0}^{1} t^{k}(1-t)^{m-k} G^{\prime}(t) d t
$$

It follows easily from Theorem 4 and the properties of $G^{\prime}(t)$ and $A(t)$ that

$$
\int_{0}^{1} t^{k}(1-t)^{m-k} G^{\prime}(t) d t \sim \int_{0}^{1} t^{k}(1-t)^{m-k} A(t) d t
$$

Therefore it is enough to show that, for all $k=1, \ldots,\lfloor m / 2\rfloor$,

$$
A\left(\frac{k}{m}\right) \sim(m+1)\left(\begin{array}{l}
m \\
k
\end{array}\right) \int_{0}^{1} t^{k}(1-t)^{m-k} A(t) d t
$$

Write $I(m, k)$ for the expression on the right. $I(m, k)$ would decrease if we only integrated on the interval $[\mathrm{k} / \mathrm{m}, 1]$, and, since $A(t)$ is increasing, it would decrease further if we replaced $A(t)$ by $A(k / m)$. This shows that

$$
I(m, k) \geq A\left(\frac{k}{m}\right)(m+1)\left(\begin{array}{l}
m \\
k
\end{array}\right) \int_{k / m}^{1} t^{k}(1-t)^{m-k} d t \geq c_{2} A\left(\frac{k}{m}\right)
$$

For the last inequality it should be proved that

$$
(m+1)\left(\begin{array}{l}
m \\
k
\end{array}\right) \int_{k / m}^{1} t^{k}(1-t)^{m-k} d t \geq c_{2}>0
$$

for all $k=1, \ldots,\lfloor m / 2\rfloor$ and for all large enough $m$. This can be done as follows. 
The integrand is maximal at $t=k / m$ and decreases on $[k / m, 1]$. So, for any $T \in[k / m, 1]$,

$$
\int_{k / m}^{1} t^{k}(1-t)^{m-k} d t \geq\left(T-\frac{k}{m}\right) T^{k}(1-T)^{m-k}
$$

Choosing $T=(k+\sqrt{k}) / m$ gives a good lower bound for the integral. We omit the technical computations.

For the other inequality we observe that $A(t) \leq A(k / m)$ when $t \leq k / m$. From property 2 above,

$$
A(t) \leq c_{15}\left(\frac{t m}{k}\right)^{2} A\left(\frac{k}{m}\right)
$$

when $t \geq k / m$. This gives

$$
\begin{aligned}
I(m, k) & \leq(m+1)\left(\begin{array}{l}
m \\
k
\end{array}\right) A\left(\frac{k}{m}\right)\left[\int_{0}^{k / m} t^{k}(1-t)^{m-k} d t+\int_{k / m}^{1} t^{k}(1-t)^{m-k} c_{15}\left(\frac{t m}{k}\right)^{2} d t\right] \\
& \leq A\left(\frac{k}{m}\right)(m+1)\left(\begin{array}{l}
m \\
k
\end{array}\right)\left[\int_{0}^{1} t^{k}(1-t)^{m-k} d t+c_{15} \frac{m^{2}}{k^{2}} \int_{0}^{1} t^{k+2}(1-t)^{m-k} d t\right]
\end{aligned}
$$

and this is less than $c_{3} A(k / m)$ by (9).

\subsection{Higher Dimensions}

We mention a possible generalization to the case $d>2$. In this case define

$$
G(t)=P\left[F\left(x_{1}, \ldots, x_{d}\right) \leq t\right]
$$

where $F\left(x_{1}, \ldots, x_{d}\right)$ is the probability content of the half-space on the right-hand side of aff $\left(x_{1}, \ldots, x_{d}\right)$. Here $x_{1}, \ldots, x_{d}$ are independent random points from $P$ (on $R^{d}$ ). Formula (2) is replaced by its directed version:

$$
2\left(\begin{array}{l}
n \\
d
\end{array}\right)\left(\begin{array}{c}
n-d \\
k
\end{array}\right) \int_{0}^{1} t^{k}(1-t)^{n-d-k} d G(t)
$$

Let $P$ be the uniform distribution on a convex body $K \subset R^{d}$. Define $v$ and $A(t)$ as in (3). It is proved in [3] that $G(t) \sim t^{d-1} A(t)$ for any convex body $K \in R^{d}$ but what we need here is the behavior of the derivative of $G$. This does not seem to be easy to establish and we could only settle the case when $K$ is smooth (say $\mathscr{C}^{3}$ ) 
with the Gauss-Kronecker curvature bounded away from zero and infinity. In this case we can prove

$$
G^{\prime}(t) \sim t^{d-2} A(t)
$$

and so

$$
E_{d}(k, n) \sim\left(\begin{array}{l}
n \\
d
\end{array}\right)\left(\begin{array}{c}
n-d \\
k
\end{array}\right) \int_{0}^{1} t^{k+d-2}(1-t)^{n-d-k} A(t) d t
$$

It is known that, for a $\mathscr{C}^{3}$ convex body $K, A(t) \sim t^{2 /(d+1)}$ which gives

$$
E_{d}(k, n) \sim k^{d-2+2 /(d+1)} n^{1-2 /(d+1)}
$$

in view of (9). This shows, again, that $E_{d}(k, n)$ behaves like the expected number of facets (or vertices, edges, etc.) of the random polytope inscribed in $K$ when $k$ is constant and like $n^{d-1}$ when $k>c n$. This is probably true for all convex bodies $K \subset R^{d}$, not only for the $\mathscr{C}^{3}$ ones.

\section{A Distribution with Many Halving Lines}

Erdös et al. [12] exhibited a set $T_{i}$ of $n_{i}=3 \cdot 2^{i}$ points which has at least $c n_{i} \log n_{i}$ halving segments. We use this example to construct distributions $P$ for which $E_{2}((n-2) / 2, n) \geq c_{8} n \log n$. First we review the example of [12] and point out some new features that are needed for the analysis.

The example is sequential. At step $i=1$ there are $n_{1}=6$ points; three are vertices of an equilateral triangle and three are on rays from the center through these vertices, as in Fig. 4(a). Clearly, there are $h_{1}=6$ halving segments. To form $T_{2}$,

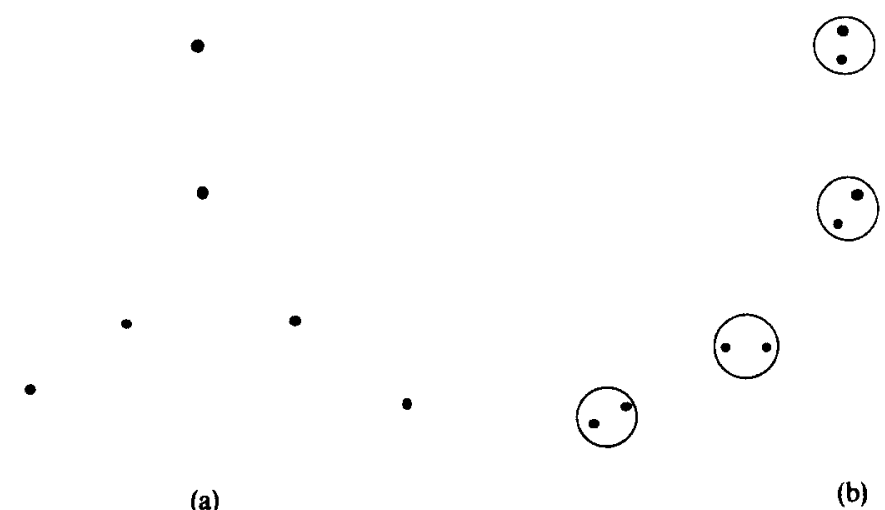

Fig. 4. Sets $T_{1}$ and $T_{2}$. 


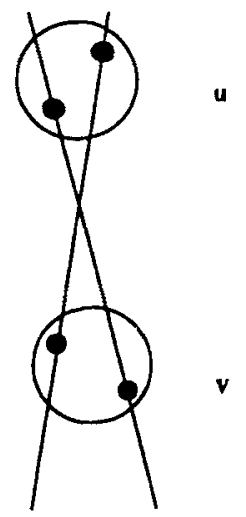

Fig. 5. Halving pair $u, v \in T_{i}$ begets two pairs in $T_{i+1}$.

each point $u \in T_{1}$ splits into two close points $u_{1}, u_{2}$ which are positioned so they define a halving line, as in Fig. 4(b). In addition each pair $u, v$ that defined a halving line in $T_{1}$ now defines two halving lines, as shown in Fig. 4(b) (see also Fig. 5). This gives $n_{2}=3 \cdot 2^{2}=12$ points with $h_{2}=18$.

In general, $T_{i}$ has $n_{i}=3 \cdot 2^{i}$ points. It is shown in [12] that each point $u \in T_{i}$ may be replaced by two close points $u_{1}, u_{2}$ which can be positioned so that:

1. $u_{1} u_{2}$ is a halving segment in $T_{i+1}$.

2. If $u v$ was a halving segment in $T_{i}$, two new halving lines are formed from $u_{1}, u_{2}, v_{1}, v_{2}$ (see Fig. 5).

If $h_{i}$ denotes the number of halving segments in $T_{i}$, properties 1 and 2 , respectively, show that

$$
h_{i+1}=n_{i}+2 h_{i}, \quad h_{1}=6,
$$

a recurrence with solution $h_{i}=3 \cdot 2^{i-1}(i+1)$.

To describe our construction we need to know $f_{i}(j)$, the number of $j$-segments in $T_{i}, j=0,1, \ldots, n_{i} / 2-1$. We have used $h_{i}$ for $f_{i}\left(n_{i} / 2-1\right)$ and we write $h_{i}^{-}=$ $f_{i}\left(n_{i} / 2-2\right)$ for the number of segments that are one-less-than-halving. From Fig. 5 , if $u v$ was a $j$-segment in $T_{i}$, then the four segments $u_{1} v_{1}, u_{1} v_{2}, u_{2} v_{1}, u_{2} v_{2}$ form two $(2 j+1)$-segments and a $2 j$-segment and a $(2 j+2)$-segment in $T_{i+1}$. However, when $j=n_{i} / 2-1$, the $2 j$-segment and the $(2 j+2)$-segment are both one-less-thanhalving. Therefore $f_{i+1}(0)=f_{i}(0)$ and

$$
\begin{aligned}
f_{i+1}(2 j) & =f_{i}(j)+f_{i}(j-1), \quad j=1, \ldots, \frac{n_{i}}{2}-2, \\
f_{i+1}(2 j+1) & =2 f_{i}(j), \quad j=0,1, \ldots, \frac{n_{i}}{2}-1,
\end{aligned}
$$




$$
\begin{aligned}
f_{i+1}(2 j) & =2 f_{i}(j)+f_{i}(j-1), \quad j=\frac{n_{i}}{2}-1, \\
f_{i+1}(2 j+1) & =2 f_{i}(j)+n_{i}, \quad j=\frac{n_{i}}{2}-1 .
\end{aligned}
$$

Equation (19) is the recurrence for $h_{i}$, while (18) gives

$$
h_{i+1}^{-}=2 h_{i}+h_{i}^{-}, \quad h_{1}^{-}=6,
$$

a recursion solved by $h_{i}^{-}=3 \cdot 2^{i}(i-1)+6$.

A convenient way to represent $f_{i}$ is via the continuous function $g_{i}$ on $\left[0, \frac{1}{2}\right]$ with values $g_{i}(0)=0$ and

$$
g_{i}\left(\frac{j+1}{n_{i}}\right)=\frac{f_{i}(j)}{n_{i}}, \quad j=0,1, \ldots, \frac{n}{2}-1,
$$

and linear between the points $j / n_{i}$. Evaluating (19) for $j=n_{i} / 2-1$ shows that $g_{i}\left(\frac{1}{2}\right)=h_{i} / n_{i}=(i+1) / 2$ and for $j=n_{i} / 2-2$, that $g_{i}\left(\frac{1}{2}-1 / n_{i}\right)=h_{i}^{-} / n_{i} \geq i-1$. From (17) and (18), for $j \leq n_{i} / 2-2, g_{i+1}\left((j+1) / n_{i}\right)=g_{i}\left((j+1) / n_{i}\right)$ and this implies $g_{i+k}\left((j+1) / n_{i}\right)=g_{i}\left((j+1) / n_{i}\right)$. Therefore, for $t \leq t_{i}=\frac{1}{2}-1 / n_{i}, g_{i+k}(t)=g_{i}(t)$, by the linearity of $g_{i}$. These relations allow the computation of all values of $f_{i}(j)$.

We now make $T_{i}$ into a set $S_{i}$ of positive area by replacing each point $x \in T_{i}$ by the disk centered at $x$ with radius $\varepsilon_{i}$, which may be chosen small enough so that the disks are in general position (no three stabbed by a line). It is not surprising that:

Lemma 3. If $P_{i}$ is the uniform distribution on $S_{i}$, then $E_{2}((n-2) / 2, n)=\Omega(n \log n)$ as long as an $<n_{i}<b n$, for fixed $0<a<b<\infty$.

Proof. We have, according to (2),

$$
\begin{aligned}
E_{2}\left(\frac{n-2}{2}, n\right) & =\left(\begin{array}{l}
n \\
2
\end{array}\right)\left(\begin{array}{c}
n-2 \\
n / 2-1
\end{array}\right) \int_{0}^{1 / 2} 2[t(1-t)]^{n / 2-1} d G(t) \\
& \geq\left(\begin{array}{l}
n \\
2
\end{array}\right) c_{16} \frac{2^{n}}{\sqrt{n}} \int_{1 / 2-1 / \sqrt{n_{i}}}^{1 / 2} 2[t(1-t)]^{n / 2-1} d G(t) \\
& \geq\left(\begin{array}{l}
n \\
2
\end{array}\right) c_{16} \frac{2^{n}}{\sqrt{n}} \int_{1 / 2-1 / \sqrt{n_{i}}}^{1 / 2} \frac{c_{17}}{2^{n}}\left[1-\frac{4}{n_{i}}\right]^{n / 2-1} d G(t) \\
& \leq c_{18} n^{3 / 2} e^{-2\left(n / n_{i}\right)} \int_{1 / 2-1 / \sqrt{n_{i}}}^{1 / 2} d G(t) \\
& =c_{18} n^{3 / 2} e^{-2\left(n / n_{i}\right)}\left[G\left(\frac{1}{2}\right)-G\left(\frac{1}{2}-\frac{1}{\sqrt{n_{i}}}\right)\right] .
\end{aligned}
$$


Now let $x$ and $y$ be two points chosen independently and randomly according to $P_{i}$. Write $D$ for the event that $x, y$ are not in the same disk; clearly, $\operatorname{Prob}[D]=$ $1-1 / n_{i}$. We have

$$
\begin{aligned}
G\left(\frac{1}{2}\right)-G\left(\frac{1}{2}-\frac{1}{\sqrt{n_{i}}}\right) & =\operatorname{Prob}\left[F(x y) \in\left[\frac{1}{2}-\frac{1}{\sqrt{n_{i}}}, \frac{1}{2}\right]\right] \\
& \geq \operatorname{Prob}\left[F(x y) \in\left[\frac{1}{2}-\frac{1}{\sqrt{n_{i}}}, \frac{1}{2}\right] \mid D\right] \operatorname{Prob}[D] \\
& \geq\left(1-\frac{1}{n_{i}}\right)_{j=n_{i} / 2-\sqrt{n_{i}}} \frac{f_{i}\left(\begin{array}{c}
n_{i} \\
2
\end{array}\right)}{n_{i} / 2-1} \\
& \geq\left(n_{i}-1\right)\left(\begin{array}{c}
n_{i} \\
2
\end{array}\right)^{-1} \sum_{j=n_{i}\left(1 / 2-1 / \sqrt{n_{i}}\right)}^{n_{i} / 2-1} g_{i}\left(\frac{j+1}{n_{i}}\right) .
\end{aligned}
$$

Note that $t_{i / 2}=\frac{1}{2}-1 / m_{i / 2}>\frac{1}{2}-1 / \sqrt{n_{i}}$ and that there are $2^{i-k-1}$ values of $j$ such that $t_{k} \leq(j+1) / n_{i}<t_{k+1}$. Therefore,

$$
\begin{aligned}
G\left(\frac{1}{2}\right)-G\left(\frac{1}{2}-1 \sqrt{n_{i}}\right) & \geq \frac{2}{n_{i}} \sum_{k=i / 2}^{i-1} \sum_{j=t_{k}}^{k_{k+1}-1} g_{i}\left(\frac{j+1}{n_{i}}\right) \geq \frac{2}{n_{i}} \sum_{k=i / 2}^{i-1} g_{i}\left(t_{k}\right) 2^{i-k-1} \\
& \geq \frac{2}{n_{i}} \sum_{k=i / 2}^{i-1}(k-1) 2^{i-k-1} \geq\left(\frac{2}{n_{i}}\right) \frac{i}{4} 2^{i / 2}
\end{aligned}
$$

Combined with (21),

$$
E_{2}\left(\frac{n-2}{2}, n\right) \geq c_{18} n^{3 / 2} e^{-2\left(n / n_{i}\right)} c_{19} \frac{\log n_{i}}{\sqrt{n_{i}}}
$$

and so $E_{2} \geq c_{7} n \log n$.

On the other hand, it is straightforward to show that, as $n \rightarrow \infty$, the expected number of halving segments for $n$ points chosen from $P_{i}$ is $O(n)$. The argument is a simple calculation like the one in (8) using the fact that $d G$ is bounded as $n$ increases.

Next we show that there is a single distribution for which $E_{2}$ grows at a superlinear rate. Assume that a sequence $w_{m} \rightarrow 0$ is given. We construct an absolutely continuous distribution $P$ for which $E_{2}((n-2) / 2, n) \geq c_{7} w_{n} n \log n$ for any $n$. We use the same sequence of sets $T_{i}$ and system of disks $S_{i}$ as before with the nesting condition that $S_{i} \supset S_{i+1}$. This can be achieved if, in each step, the radii of the disks are small enough.

Define $P$ by requiring that $P\left(S_{i}\right)=m_{i}$ with every disk in $S_{i}$ having probability 
content $m_{i} / n_{i}, i=1,2, \ldots ; m_{i}$ is specified later. Clearly, $m_{1}=1$ must hold and as $S_{i} \supset S_{i+1}$ we have $m_{i} \geq m_{i+1}$. If $m_{i}>m_{i+1}$ we define $P$, restricted to $S_{i} \backslash S_{i+1}$, to be uniform on $S_{i} \backslash S_{i+1}$. $P$ is a probability measure for every sequence $1=$ $m_{1} \geq m_{2} \geq \cdots$ of positive numbers.

Arguing as in (21) we see that

$$
E\left(\frac{n-2}{2}, n\right) \geq c_{20} n^{3 / 2}\left[G\left(\frac{1}{2}\right)-G\left(\frac{1}{2}-\frac{1}{\sqrt{n}}\right)\right]
$$

As in the proof of Lemma 3 we let $x, y$ denote a random pair of points distributed according to $P$. Define $i$ by requiring $n_{i} \leq n<n_{i+1}$. Let $D_{i}$ denote the event that both $x$ and $y$ are in $S_{i}$ but belong to different disks of $S_{i}$. Clearly,

$$
\operatorname{Prob}\left[D_{i}\right]=m_{i}^{2}\left(1-\frac{1}{n_{i}}\right)
$$

The previous computation applies now in the following way:

$$
\begin{aligned}
G\left(\frac{1}{2}\right)-G\left(\frac{1}{2}-\frac{1}{\sqrt{n}}\right) & \geq \operatorname{Prob}\left[F(x y) \in\left[\frac{1}{2}-\frac{1}{\sqrt{n_{i}}}, \frac{1}{2}\right] \mid D_{i}\right] \operatorname{Prob}\left[D_{i}\right] \\
& \geq \frac{2}{n_{i}} \frac{i}{4} 2^{i / 2} m_{i}^{2} \geq c_{7} m_{i}^{2} n \log n .
\end{aligned}
$$

If we choose $m_{i}=1$ for all $i$, then $P$ is a probability distribution, with support $\bigcap S_{i}$ and having $E_{2} \sim n \log n$. This distribution is concentrated in a small set. If we choose a decreasing sequence $m_{i}$ slowly tending to zero, then $P$ is an absolutely continuous measure and $E_{2} \geq m_{n}^{2} n \log n$.

\section{Acknowledgments}

The authors are grateful to Günter Rote who pointed out an error in an earlier version, and made many useful suggestions for the current one. A referee made suggestions for improving the presentation.

\section{References}

1. N. Alon and E. Györi. The number of small semispaces of a finite set of points. J. Combin. Theory Ser. A, 41:154-157, 1986.

2. B. Aronov, B. Chazelle, H. Edelsbrunner, L. Guibas, M. Sharir, and R. Wenger. Points and triangles in the plane and halving planes in space. Discrete Comput. Geom., 6:435-442, 1991.

3. I. Bárány. Intrinsic volumes and $f$-vectors of random polyopes. Math. Ann., 285:671-699, 1989.

4. 1. Bárány and Z. Füredi. On the shape of the convex hull of random points. Probab. Theory Rel. Fields, 77:231-240, 1988. 
5. I. Bárány, Z. Füredi, and L. Lovász. On the number of halving planes in $R^{3}$. Combinatorica, 10:175-193, 1990.

6. I. Bárány and D. Larman. Convex bodies, economic cap coverings, random polytopes. Mathematika, 35:274-291, 1988.

7. I. Bárany and W. Steiger. On the expected number of k-sets. Proceedings of the Second Canadian Conference on Computational Geometry, 1990, pp. 55-59.

8. L. Danzer, B. Grunbaum, and V. Klee. Helly's Theorem and Its Relatives. Proceedings of Symposia in Pure Mathematics, Vol. VII. American Mathematical Society, Providence, RI, 1963.

9. T. Dey and H. Edelsbrunner. Counting simplex crossings and halving hyperplanes. Preprint, University of Illinois, 1992.

10. H. Edelsbrunner. Algorithms in Combinatorial Geometry. Springer-Verlag, Berlin, 1987.

11. H. Edelsbrunner and E. Welzl. On the number of line separations of a finite set in the plane. J. Combin. Theory Ser. A, 38:15-29, 1985.

12. P. Erdős, L. Lovász, A. Simmons, and E. Strauss. Dissection graphs of planar point sets. In $A$ Survey of Combinatorial Theory (J. Srivastava et al., eds.), pp. 139-149. North-Holland, Amsterdam, 1973.

13. J. E. Goodman and R. Pollack. On the number of k-sets of a set of $n$ points in the plane. J. Combin. Theory Ser. A, 36:101-104, 1984.

14. Chi-Yuan Lo, J. Matoušek, and W. Steiger. Ham-sandwich cuts in $R^{d}$. Proceedings of the 24th ACM Symposium on Theory of Computing, 1992, pp. 539-545.

15. L. Lovász. On the number of halving lines. Ann. Univ. Sci. Budapest. Eötvös Sect. Math., $14: 107-108,1971$.

16. R. Miles. Isotropic random simplices. Adv. in Appl. Probab., 3:353-382, 1971.

17. J. Pach, W. Steiger, and E. Szemerédi. An upper bound on the number of planar $K$-sets. Discrete Comput. Geom., 7:109-123, 1992.

18. J. Pach and J. Töröcsik. Layout of rooted trees. Manuscript, 1991.

19. A. Rényı and R. Sulanke. Über die konvexe hülle von $n$ zufällig gewählten punkten, I, II. $Z$. Wahrsch. Verw. Gebiete, 2:75-84, 1963; 3:138-147, 1964.

20. L. Santaló. Integral Geometry and Geometric Probability. Addison-Wesley, Reading, MA, 1976.

21. C. Schütt and E. Werner. The convex floating body. Math. Scand., 66:275 290, 1990.

22. E. Welzl. More on $k$-sets of finite sets in the plane. Discrete Comput. Geom., 1:95-100, 1986.

23. R. T. Živaljević and S. T. Vrećica. The colored Tverberg's problem and complexes of injective functions. J. Combin. Theory Ser. A, 61:309-318, 1992.

Received June 10, 1992, and in revised form August 25, 1993. 